\title{
Femtojoule, Femtosecond, All-Optical Switching in Integrated Lithium Niobate Photonics
}

\author{
Qiushi Guo $^{1 *}$, Ryoto Sekine ${ }^{1 *}$, Luis Ledezma ${ }^{1,2}$, Devin J. Dean ${ }^{3}$, Rajveer Nehra ${ }^{1}$, \\ Arkadev Roy ${ }^{1}$, Alireza Marandi ${ }^{1}$ \\ ${ }^{1}$ Department of Electrical Engineering, California Institute of Technology, Pasadena, California 91125, USA \\ 2 Jet Propulsion Laboratory, California 91109, USA \\ ${ }^{3}$ School of Applied and Engineering Physics, Cornell University, Ithaca, New York 91125, USA \\ *These authors contribute equally to this work \\ marandi@caltech.edu
}

\begin{abstract}
We introduce and demonstrate an integrated nonlinear splitter in quasi-phase matched lithium niobate nanophotonic waveguide featuring femtojoule all-optical switching with instantaneous response and $8.5 \mathrm{~dB}$ extinction, opening opportunities for on-chip ultrafast energy-efficient all-optical information processing. () 2021 The Author(s)
\end{abstract}

\section{Main text}

Optical nonlinear functions are crucial for a wide range of on-chip ultrafast photonic applications, such as optical switching/gating [1], photonic neural networks [2], and on-chip ultrafast light sources [3]. However, a major challenge to realize on-chip optical nonlinear functions comes from the exceedingly weak nonlinearities available in most photonic platforms. Therefore, implementing nonlinear functions typically require large driving energies of picojoules to nanojoules per pulse, which is a barrier to most applications. Although the optical nonlinearity can be enhanced by resonant structures [1], this comes at the cost of an unavoidable trade-off in switching speed. Here, we demonstrate a chip-scale integrated nonlinear splitter device, which exhibits all-optical switching with instantaneous optical response, large extinction ratio and ultralow operational energy down to femtojoules.

Central to our device is the strong quadratic $\chi^{(2)}$ nonlinearity and highly-efficient nonlinear frequency conversion $\left(>1100 \% / \mathrm{W}-\mathrm{cm}^{2}\right)$ in quasi-phase matched dispersion-engineered lithium niobate (LN) nanophotonic waveguides [4]. Figure 1a illustrates the integrated nonlinear splitter and its operating principle, which is similar to the previously implemented nonlinear mirrors with bulk crystals [5]. The device is composed of two periodically poled LN (PPLN) waveguides separated by an unpoled region (poling defect) with a length $L_{\mathrm{D}}$ equal to the poling periodicity, and a directional coupler. When the input fundamental harmonic $(\mathrm{FH})$ enters the device from the left, due to second-harmonic generation ( $\mathrm{SHG}$ ) at the beginning of the waveguide, some of the FH gets converted to the second harmonic ( $\mathrm{SH}$ ) and part of the remaining FH is evanescently coupled to "output 2" by the directional coupler. The poling defect in the middle of the waveguide causes a $180^{\circ}$ phase shift between the FH and SH switching the SHG process to the optical parametric amplification (OPA) in the second half of the PPLN waveguide, through which the SH will be converted back into FH. In the "on" state with high pulse energies (Fig. $1 \mathrm{~b}$ left), the device favors transmission of the FH to "output 1" since more energy can be stored in (i.e. converted to) the SH, which is free from the outcoupling. On the other hand, at low input energies (or the "off" state shown in Fig. 1b right), most of the input is not converted to SH, and hence will be directed to "output 2". Fig. 1c shows the simulated transmission of the $2.09 \mu \mathrm{m} 35$ fs pulses from the input to "output 1 " as a function of input average power and pulse energy. For a nonlinear splitter device with splitting ratio of 10:90, the transmission of $2.09 \mu \mathrm{m}$ pulses increases dramatically from $10 \%$ to more than $60 \%$ within $1 \mathrm{pJ}$ energy range (more than $8.5 \mathrm{~dB}$ extinction ratio). Note that the required pulse energy for achieving $3 \mathrm{~dB}$ extinction ratio is less than $400 \mathrm{fJ}$.

Our device is fabricated on a $700 \mathrm{~nm}$ thick X-cut $\mathrm{MgO}$ doped $\mathrm{LN}$ thin film on a 2- $\mu \mathrm{m}$-thick silicon dioxide layer on top of a LN substrate. The fabricated nonlinear splitter devices are shown in Fig. 2a. The inverted domains and the poling defect can be seen in the colorized two-photon microscope image (Fig. 2b). We characterize the nonlinear behavior of the device using 35-fs-long pulses at $2.09 \mu \mathrm{m}$ from a synchronously pumped degenerate optical parametric oscillator (OPO) with a repetition frequency of $250 \mathrm{MHz}$. The experimentally measured spectra from the waveguide output port 1 are shown in Fig. 2c, in which spectral features at $2090 \mathrm{~nm}, 1045 \mathrm{~nm}, 696 \mathrm{~nm}$ and $522 \mathrm{~nm}$ corresponds to the $\mathrm{FH}, \mathrm{SH}$, the sum frequency generation between $\mathrm{FH}$ and $\mathrm{SH}$ and higher harmonic, respectively. As shown in Fig. 2d, the transmittance from input to output port 1 shows a clear increase by a factor of 5 as input pulse energy increases from 40 to 400 fJ. Our integrated nonlinear splitter can address the long-standing 
challenge of achieving low-energy high-speed nonlinear functions in integrated photonics with diverse potential applications. As an on-chip ultrafast all-optical switch, our device features the state-of-the-art switching energy as low as hundreds of $\mathrm{fJ}$ and a large modulation depth more than $8.5 \mathrm{~dB}$. The nonlinear transmission characteristic can also be utilized as the nonlinear activation function required to construct artificial photonic neural networks. Due to the instantaneous nature of the quadratic nonlinearity, the switching speed of the device is limited by the pulse length, which can be in the range of 10 s of femtosecond in the current device. Lastly, the nonlinear splitter device can be tailored as an integrated saturable absorber for low pulse energy passive mode locking.
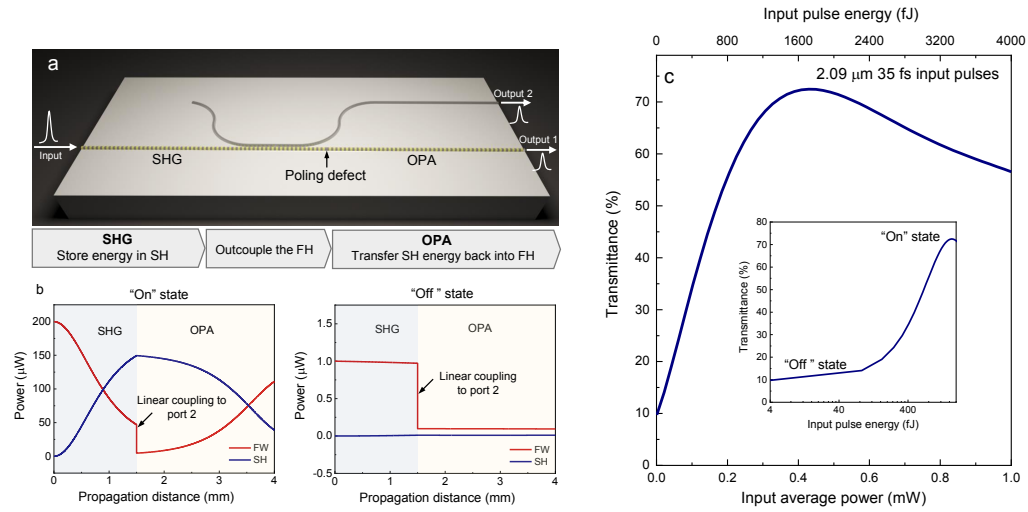

Fig. 1. (a) Schematic of nonlinear splitter and its basic operation. (b) Simulated evolution of the FH and SH optical power along the PPLN waveguide for the "on" and the "off" states. The simulated device has 1.5-mm-long SHG region, 2.5-mm-long OPA region and 90\% outcoupling of FH. (d) Simulation results of power at "output 1 " at $2.09 \mu \mathrm{m}$ as a function of input power for 2.09- $\mu \mathrm{m}$, 35-fs-long pulses. Inset: corresponding power-dependent transmittance (input to output 1)
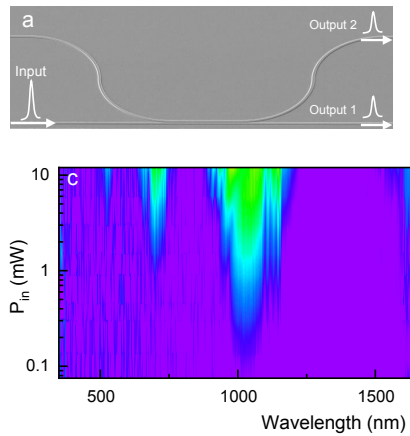
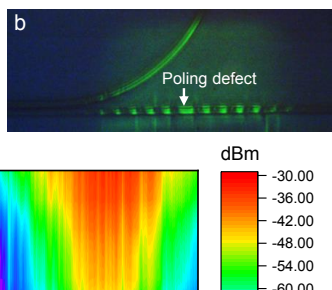

--54.00
-60.00
-66.00

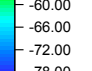

-78.00
--84.00

$-84.00$

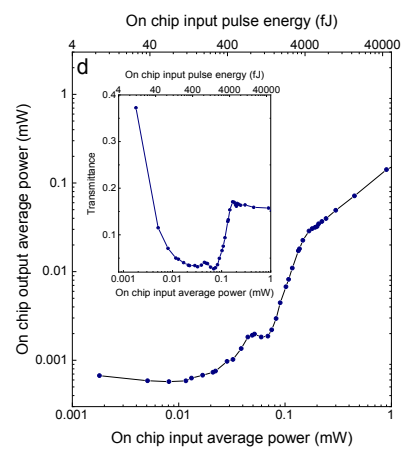

Fig. 2. (a) SEM image of the fabricated device. (b) Two-photon microscope image showing the inverted domains and the poling defect along the waveguides. (c) Measured power spectral density at "output 1" from the chip as a function of input average power. (d) Measured power of FH at “output 1 ” for 2.09- $\mu \mathrm{m}, 35$-fs-long pulses. Inset: power dependent transmission (input to output 1).

\section{References}

1. K. Nozaki, T. Tanabe, A. Shinya, S. Matsuo, T. Sato, H. Taniyama, and M. Notomi, "Sub-femtojoule alloptical switching using a photonic-crystal nanocavity," Nat. Photonics 4, 477-483 (2010).

2. P. R. Prucnal and B. J. Shastri, Neuromorphic Photonics (CRC Press, 2017).

3. A. W. Fang, B. R. Koch, K.-G. Gan, H. Park, R. Jones, O. Cohen, M. J. Paniccia, D. J. Blumenthal, and J. E. Bowers, "A racetrack mode-locked silicon evanescent laser," Opt. Express 16, 1393-1398 (2008).

4. M. Jankowski, C. Langrock, B. Desiatov, A. Marandi, C. Wang, M. Zhang, C. R. Phillips, M. Lončar, and M. Fejer, "Ultrabroadband nonlinear optics in nanophotonic periodically poled lithium niobate waveguides," Optica 7, 40-46 (2020).

5. K. Stankov and J. Jethwa, "A new mode-locking technique using a nonlinear mirror," Opt. Commun. 66, 41-46 (1988). 